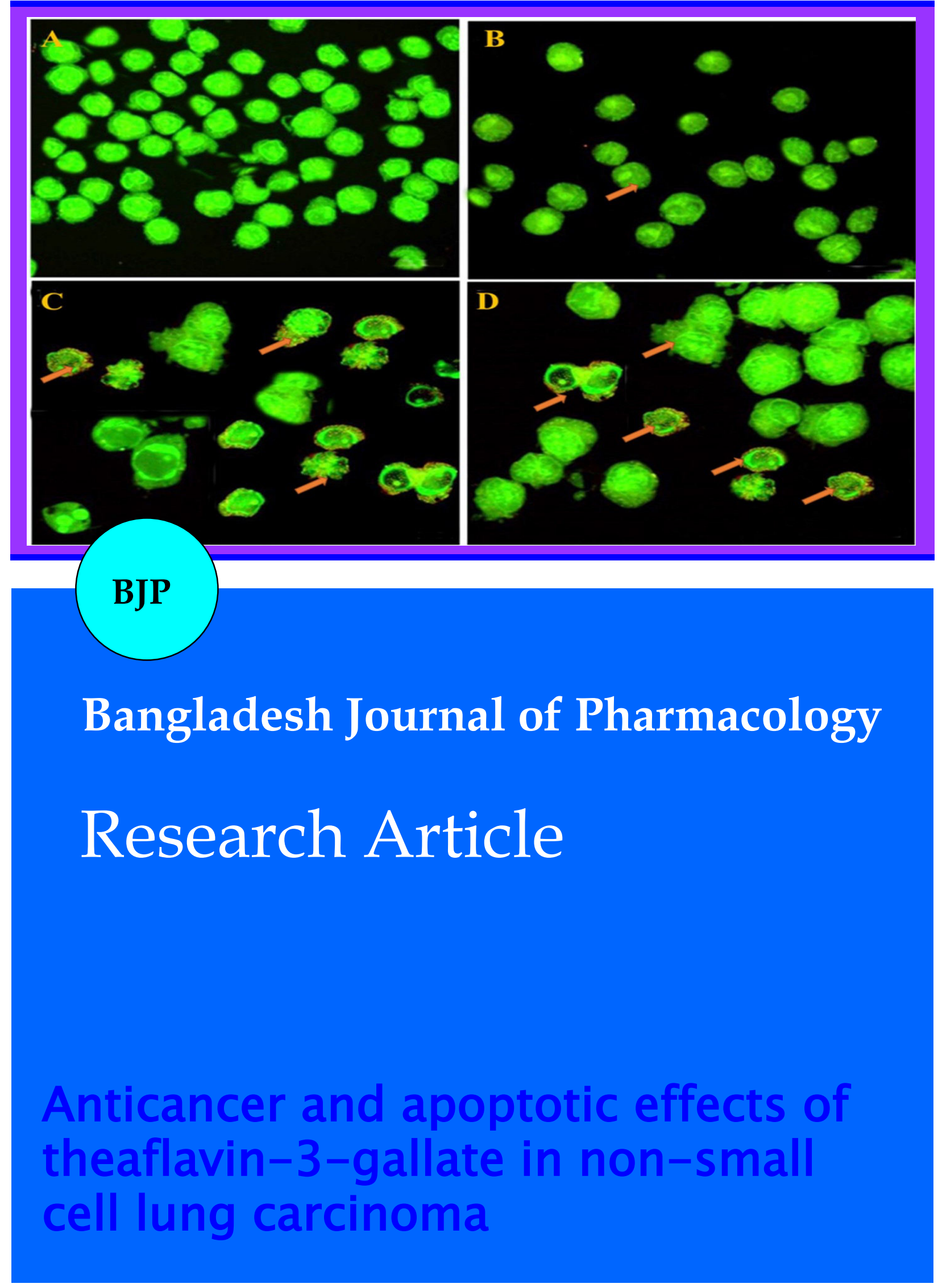




\title{
Anticancer and apoptotic effects of theaflavin-3-gallate in non-small cell lung carcinoma
}

\author{
Da-Wei Li', Long Meng², Kui-Xing Zhang1 and Wei-Ke Zhang3

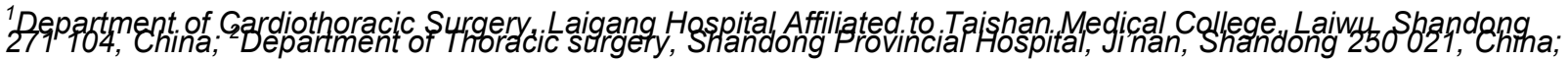 \\ ${ }^{3}$ Department of Oncologv. Laigang Hospital Affiliated to Taishan Medical College, Laiwu. Shandong 271 104. China.
}

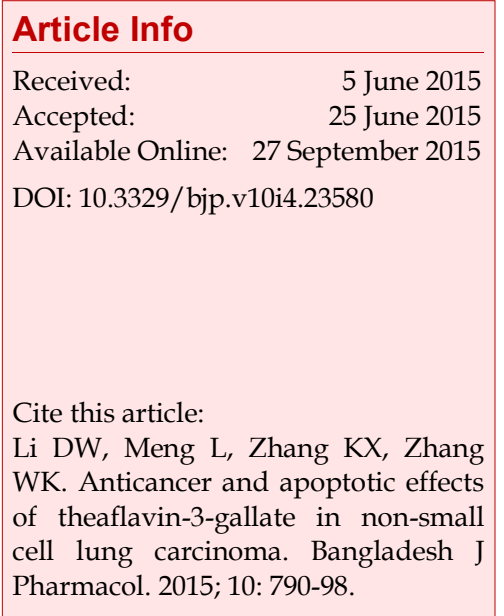

\begin{abstract}
The objective was to determine the antiproliferative and apoptotic effects of theaflavin-3-gallate in human non-small cell lung cancer cells (A-549) along with determining the effect on cell cycle phase distribution, cell migration and invasion. Cell viability was determined by MTT assay while as phase contrast and fluorescence microscopies were involved to study apoptotic morphological features in these cells. Flow cytometry investigated the effect of theaflavin3-gallate on cell cycle phase distribution. Theaflavin-3-gallate treatment led to a substantial cytotoxic effect in A-549 cancer cells with $\mathrm{IC}_{50}$ values of $42.1 \mu \mathrm{M}$ and $27.9 \mu \mathrm{M}$ at 24 and 48 hours respectively. Further, 80 and $160 \mu \mathrm{M}$ dose of theaflavin-3-gallate induced apoptotic features including chromatin margination and micronuclei presence. The population of cells in G2/M phase increased from $2.7 \%$ (control) to $6.8 \%, 17.2 \%$ and finally to $46.5 \%$ after treatment with 20,80 and $160 \mu \mathrm{M}$ concentration of theaflavin-3-gallate respectively indicating $\mathrm{G} 2 / \mathrm{M}$ phase cell cycle arrest.
\end{abstract}

\section{Introduction}

Cancer, which is the leading cause of death, is characterized by numerous hallmarks including uncontrolled growth and the tendency of cancer cells to migrate and invade neighboring tissues (Hanahan and Weinberg, 2011). The primary tumor ultimately transforms into a cancerous phenotype through a series of advanced physiological changes. The malignant phenotype leads to the spread of the cancers through metastasis. The invasion and development of a secondary tumor encompasses a cascade of consecutive steps, such as intravasation into the circulatory system and extravasation to a secondary site (Joyce and Pollard, 2009). All the cancer cells do not metastasize to other locations forming new cancerous tissues. Although metastases cause more than $85 \%$ of human cancer-related deaths, they are not accountable for the growth of the primary tumor (Mehlen and Puisieux, 2006).
Lung cancer, also known as pulmonary carcinoma, is the most frequently diagnosed cancer accounting for over 1.37 million deaths every year all across the globe. Lung cancer is instigated by activation of oncogenes or inactivation of tumor inhibitor genes. Mutations in the K-ras proto-oncogene are accountable for $10-25 \%$ of lung adenocarcinomas. In China, lung cancer has substituted liver cancer as the principal cause of mortality among people with malignant tumors. According to the statistics from the National Office on Tumor Cure and Prevention, about 600,000 people die of lung cancer each year in China (Alberg et al., 2005; American Cancer Society, 2014). Clinically two general classes of lung cancer have been known, non-small-cell lung carcinoma and small-cell lung carcinoma. Non-smallcell lung carcinoma is more prevalent, accounting for almost $80 \%$ of lung cancers.

There are different options for the treatment of non- 
small-cell lung carcinoma including chemotherapy by platinum complexes such as cisplatin (cis-diammine dichloroplatinum II) and carboplatin (cis-diammine [1, 1cyclobutane-dicarboxylato] platinum) (Raghavan et al., 1997; Yagoda et al., 1979; Drelichman et al., 1985; Trump et al., 1990). The chemotherapy treatment for lung cancer depends on the tumor type. Small-cell lung carcinoma is treated primarily with chemotherapy and radiotherapy (Hann and Rudin, 2008). However, long-term chemotherapy can result in drug resistance through gene mutation or DNA methylation. As such, drug resistance in cancer patients is gradually rising and anticancer drugs like paclitaxel, cisplatin, 5-fluorouracil, doxorubicin etc are becoming less effective. Due to this growing menace of drug resistance by cancer cells, there is a pressing need to design and develop novel anticancer drugs which can overcome drug resistance problems (Ma et al., 2010; Sarkar et al., 2007). Plant based natural products have been an encouraging source of such agents and around $60 \%$ of the drugs clinically used for cancer chemotherapy are directly or indirectly derived from plants.

In this study, the anticancer effects of theaflavin-3gallate on the non-small cell lung cancer cells are reported along with its effects on apoptosis, cell cycle arrest, key cellular proteins, caspase activation, cell migration and invasion.

\section{Materials and Methods}

\section{Chemicals and other reagents}

Theaflavin-3-gallate was purchased from Sigma Chemical Company (USA). For in vitro studies, theaflavin-3gallate was dissolved in dimethyl sulfoxide (DMSO) to create a stock solution $(0.1 \mathrm{~mol} / \mathrm{L})$, which was stored at $-20^{\circ} \mathrm{C}$. To prepare working solutions, the stock solution was further diluted with culture media to yield the desired theaflavin-3-gallate concentrations. Control cells were treated with an equal volume of vehicle. The DMSO concentration was kept below $0.1 \%$ in cell culture and did not show any noticeable effect on cell growth or cell death. Dulbecco's modified Eagle's medium, fetal bovine serum (FBS), penicillin-streptomycin were obtained from Hangzhou Sijiqing Biological Products Co., Ltd, China. MTT kit was obtained from Roche (USA). Annexin V-FITC-propidium iodide apoptosis detection kit was purchased from (Beyotime Institute of Biotechnology, Shanghai, China). Acridine orange and propidium iodide were purchased from Beyotime, China. All other chemicals and solvents used were of the highest purity grade. The primary antibodies including anti-cyclin B, anti-DNA degradation factor 45 kDa (DDF45), anti-p21 and anti-cytochrome c were from BD Biosciences Co. Anti-caspase-3, anti-BCL-2, anti-BCL-xL, anti-BAD, anti-BAX, anti-poly [ADPribose] polymerase 1 (PARP-1) and anti-p53 were from
Epitomics Co. (Santa Cruz Biotechnology, Inc., Santa Cruz, CA, USA). The secondary antibodies such as goat anti-mouse IgG-HRP and goat anti-rabbit IgG-HRP were from Santa Cruz Biotechnology Inc. (Cell Signaling Technology, Inc.).

\section{Cell line and culture conditions}

Human non-small cell lung cancer cell line (A-549) was purchased from the Shanghai Institute of Cell Biology, Chinese Academy of Sciences (CAS, Shanghai, China) and was kept at $37^{\circ} \mathrm{C}$ in a humidified atmosphere containing $5 \% \mathrm{CO}_{2}$. The cells were cultured in Dulbecco's modified Eagle's medium (Sigma-Aldrich, USA) supplemented with $10 \% \mathrm{v} / \mathrm{v}$ FBS, $2 \% \mathrm{v} / \mathrm{v}$ penicillin/ streptomycin and $1 \% \mathrm{v} / \mathrm{v}$ amphotericin B.

\section{Cell viability evaluation by MTT assay}

Inhibition of cell growth by theaflavin-3-gallate was evaluated by the MTT assay. Briefly, cells were plated in 96-well culture plates $\left(1 \times 10^{5}\right.$ cells/well $)$. After 24 hours incubation, cells were treated with theaflavin-3gallate $(0,5,10,20,40,80$ and $160 \mu \mathrm{M}$, eight wells per concentration) for 24 and 48 hours, MTT solution (5 $\mathrm{mg} / \mathrm{mL}$ ) was then added to each well. After 4 hours incubation, the formazan precipitate was dissolved in $100 \mu \mathrm{L}$ dimethyl sulfoxide, and then the absorbance was measured in an ELISA reader (Thermo Molecular Devices Co., Union City, USA) at $570 \mathrm{~nm}$. The cell viability ratio was calculated by the following formula:

Inhibitory ratio $(\%)=(\mathrm{OD}$ control $-\mathrm{OD}$ treated $) / \mathrm{OD}$ control $\times 100 \%$

Cytotoxicity was expressed as the concentration of theaflavin-3-gallate inhibiting cell growth by $50 \%\left(\mathrm{IC}_{50}\right.$ value).

Evaluation of A-549 cell morphology following theaflavin-3-gallate treatment

A-549 cells were plated in six-well plates (Guangzhou Jet Biofil, Guangzhou, China) at a density of $1 \times 10^{5}$ cells $/ \mathrm{mL}$ and then cultured for 24 hours to facilitate total attachment to the surface of the plates. Subsequently, the cells were subjected to treatment with several concentrations of theaflavin-3-gallate $(0,20,80$ or $160 \mu \mathrm{M})$ for 48 hours. Following drug treatment, culture plates were examined with an inverted light microscope (Nikon Corp., Tokyo, Japan) and images were captured.

\section{Acridine orange and propidium iodide nuclei staining}

Cells were analyzed with a staining method using acridine orange and propidium iodide (Sigma-Aldrich, USA), following incubation. A-549 cells were treated with various concentrations of theaflavin-3-gallate ( 0 , $20,80$ or $160 \mu \mathrm{M})$ for 48 hours. Subsequently, cells on cover slips were collected, washed twice with phosphate-buffered saline, stained with acridine 
orange/propidium iodide solution (each $25 \mu \mathrm{g} / \mathrm{mL}$ ), and inspected and photographed using a fluorescence microscope (Nikon Corp.). Images are presented as maximum intensity projection or single-plane images.

\section{Cell cycle analysis}

A-549 cells at a density of $1 \times 10^{5}$ cells $/ \mathrm{mL}$ were grown on sterile culture plate overnight, and treated with theaflavin-3-gallate at concentrations of $0,20,80$ and $160 \mu \mathrm{M}$ for 48 hours at $37^{\circ} \mathrm{C}$ and $5 \% \mathrm{CO}_{2}$. The untreated cells were grown as a negative control. The cells were harvested, washed with phosphate buffer solution and fixed in ice-cold $70 \%$ ethanol overnight at $-20^{\circ} \mathrm{C}$. The ethanol-fixed cells were pelleted, washed with ice-cold phosphate buffer solution and resuspended in staining solution containing $50 \mu \mathrm{g} / \mathrm{mL}$ propidium iodide, 100 $\mu \mathrm{g} / \mathrm{mL}$ RNase, $0.1 \%$ sodium citrate and $0.1 \%$ Triton-X100. After incubation for $30 \mathrm{~min}$, the cells were analyzed by flow cytometer (FACS Calibur instrument (BD Biosciences, San Jose, CA, USA) equipped with Cell Quest 3.3 software).

Detection of apoptosis by annexin $V$ binding assay using flow cytometry

Detection of apoptosis was performed using the FITC annexin V apoptosis detection kit. A-549 cells at a density of $1 \times 10^{5}$ cells $/ \mathrm{mL}$ were grown on sterile culture plate overnight, and treated with theaflavin-3gallate at concentrations of $0,20,80$ and $160 \mu \mathrm{M}$ for 48 hours at $37^{\circ} \mathrm{C}$. The untreated cells were grown as a negative control. The cells were harvested, washed with phosphate buffer solution and double stained with annexin $\mathrm{V}$ and propidium iodide for $20 \mathrm{~min}$ at room temperature in dark. Apoptosis was detected by FACS Calibur flow cytometer, and distribution of cell population in different quadrants was analyzed with quadrant statistics. The lower left quadrant represents viable cells, lower right quadrant represents early apoptotic cells, upper right quadrant represents late apoptotic/ secondary necrotic cells and upper left quadrant represents primary necrotic cells.

\section{Cell migration assay}

This assay was performed using a standard method (Liang et al., 2007). Cells ( $1 \times 10^{5}$ cells $\left./ \mathrm{mL}\right)$ were seeded in a 6-well plate and incubated at $37^{\circ} \mathrm{C}$ until 95 to $100 \%$ full confluent monolayer was obtained. Subsequent to 12 hours of starvation, a $100 \mathrm{~mL}$ pipette tip was used to create a straight cell-free wound. Each well was washed twice with PBS to remove any debris and then exposed to various concentrations of theaflavin-3-gallate $(0,20$, 80 and $160 \mu \mathrm{M})$ in a medium. After 48 hours of incubation, the cells were fixed and stained with $3 \%$ ethanol containing $0.5 \%$ crystal violet powder for $20 \mathrm{~min}$, and randomly chosen fields were photographed under a light microscope (inverted light microscope (Nikon Corp., Tokyo, Japan). The number of cells that migrated into the scratched area were counted and lengths of wound were determined by Image $\mathrm{J}$ (version 1.46) software.

\section{Invasion assay}

This assay was done with a 24-well plate. Matrigel (BD) coating was prepared on a polyvinylpyrrolidone-free polycarbonate filter (6 $\mathrm{mm}$ pore size). The lower chamber was filled with medium containing $10 \%$ FBS. A-549 Cells ( 1 x $10^{5}$ cells/well) were preincubated with theaflavin-3-gallate for $20 \mathrm{~min}$ at room temperature and the cell medium containing theaflavin-3-gallate was seeded onto the upper chamber wells. Following incubation for 48 hours, the filter was fixed and stained with $3 \%$ ethanol containing $0.3 \%$ crystal violet for $20 \mathrm{~min}$. The stained cells were counted under light microscope.

\section{Western blot analysis:}

A-549 cells $\left(1 \times 10^{5}\right)$ were cultured in a $10-\mathrm{cm}$ plate and incubated for 24 hours, after which the medium was removed and replaced with fresh medium or medium containing indicated doses $(20,80$ and $160 \mu \mathrm{M})$ of theaflavin-3-gallate and incubated for another 36 hours. Then the medium was removed followed by washing with phosphate buffer solution three times before detaching cells with a cell scraper and collecting by centrifuging at 15,000 rpm for $10 \mathrm{~min}$. Lysis buffer (Cell Signaling Technology, Danvers, MA, USA) was added to cell pellet of each treatment, and cells were disrupted with a sonicator for protein release, followed by centrifuging at $14,000 \mathrm{rpm}\left(4^{\circ} \mathrm{C}\right)$ for $10 \mathrm{~min}$. The protein containing supernatant was collected and stored at $-20^{\circ}$ $\mathrm{C}$ before use. Protein concentrations were determined by Bradford assay. Proteins $(40 \mu \mathrm{g})$ of each sample lysate were resolved by $14 \%$ SDS-PAGE and transferred to nitrocellulose membranes by semi-dry transfer method. After the transfer, the membranes were blocked with $1 \%$ BSA and probed with primary and HRP-conjugated secondary antibodies using SNAP i.d. protein detection system. The blots were visualized by developing the blot with chemiluminescent substrate.

\section{Statistical analysis}

All results were presented as mean \pm standard error (S.E.) from at least three independent experiments. The independent t-test was performed using SPSS statistics 17.0 to determine the statistical significance between untreated and treated groups. Results with $\mathrm{p}<0.05$ were considered as statistically significant.

\section{Results \\ Effects of theaflavin-3-gallate on proliferation of non- small cell lung cancer cells (A-549)}

Initially we demonstrated the antiproliferative activity of theaflavin-3-gallate on A-549 cells by using MTT assay. The results revealed that theaflavin-3-gallate had potent antiproliferative effects on A-549 cells. It showed 


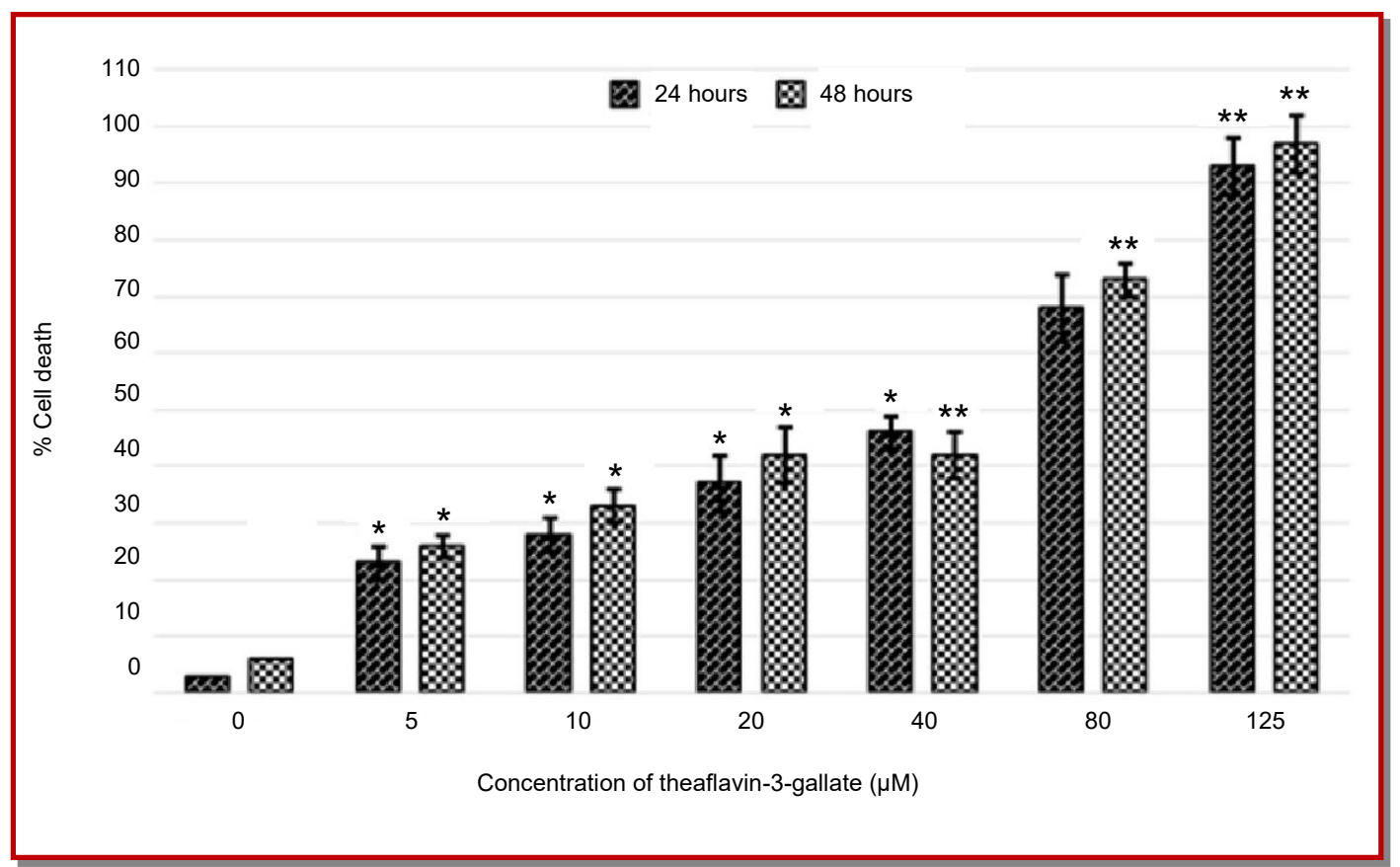

Figure 1: Cytotoxic effect of theaflavin-3-gallate in human non-small cell lung cancer cells (A-549). Data are shown as the mean \pm $\mathrm{SD}$ of three independent experiments. ${ }^{*} \mathrm{p}<0.05,{ }^{* *} \mathrm{p}<0.01$ vs $0 \mu \mathrm{M}$ (control)

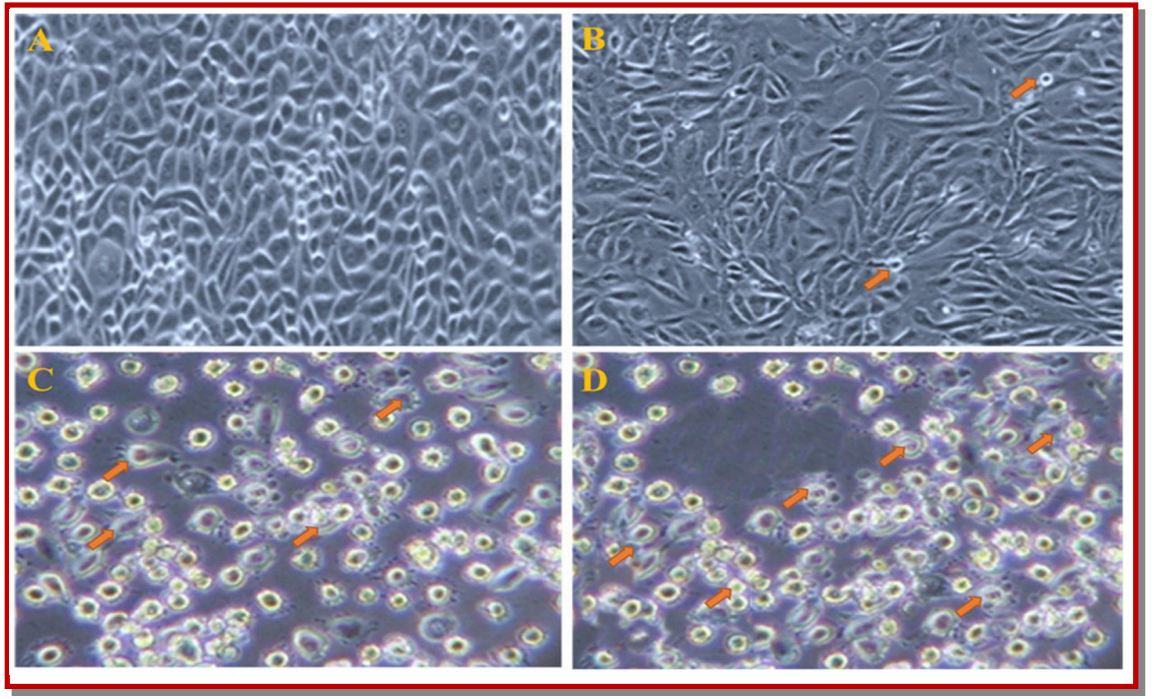

Figure 2: Morphology changes of A-549 cells after theaflavin-3-gallate treatment for 48 hours. Compound treated cells were rounded and there was no cell to cell adhesion. Arrows show membrane blebbing and rounded cells at 40x magnification. A, shows control cells with excellent cell to cell adhesion and normal cellular morphology. B, C and D represent cells treated with 20, 80 and $160 \mu \mathrm{M}$ dose of theaflavin-3-gallate

both concentration dependent as well as timedependent growth inhibitory effects against these cells (Figure 1). For determining the effectiveness of this polyphenolic compound, its $\mathrm{IC}_{50}$ value was also calculated to be $42.1 \mu \mathrm{M}$ and $27.9 \mu \mathrm{M}$ at 24 and 48 hours respectively.

\section{Morphological study of apoptosis using phase contrast microscopy}

In this study, the morphological alterations of human non-small cell lung cancer cells (A-549) untreated and treated with theaflavin-3-gallate were detected under an inverted light microscope. The most noticeable changes characteristic of apoptosis were observed in the treated cells that include the detachment of the cells from substratum, cell shrinkage. As shown by inverted light microscopy, the untreated control cells were uniformly scattered on the substratum. Decrease in the cell population was seen with the increase in the theaflavin3-gallate concentration. As can be seen in Figure 2, untreated A-549 cells appeared as densely packed and organized multilayers, whereas after incubation with 

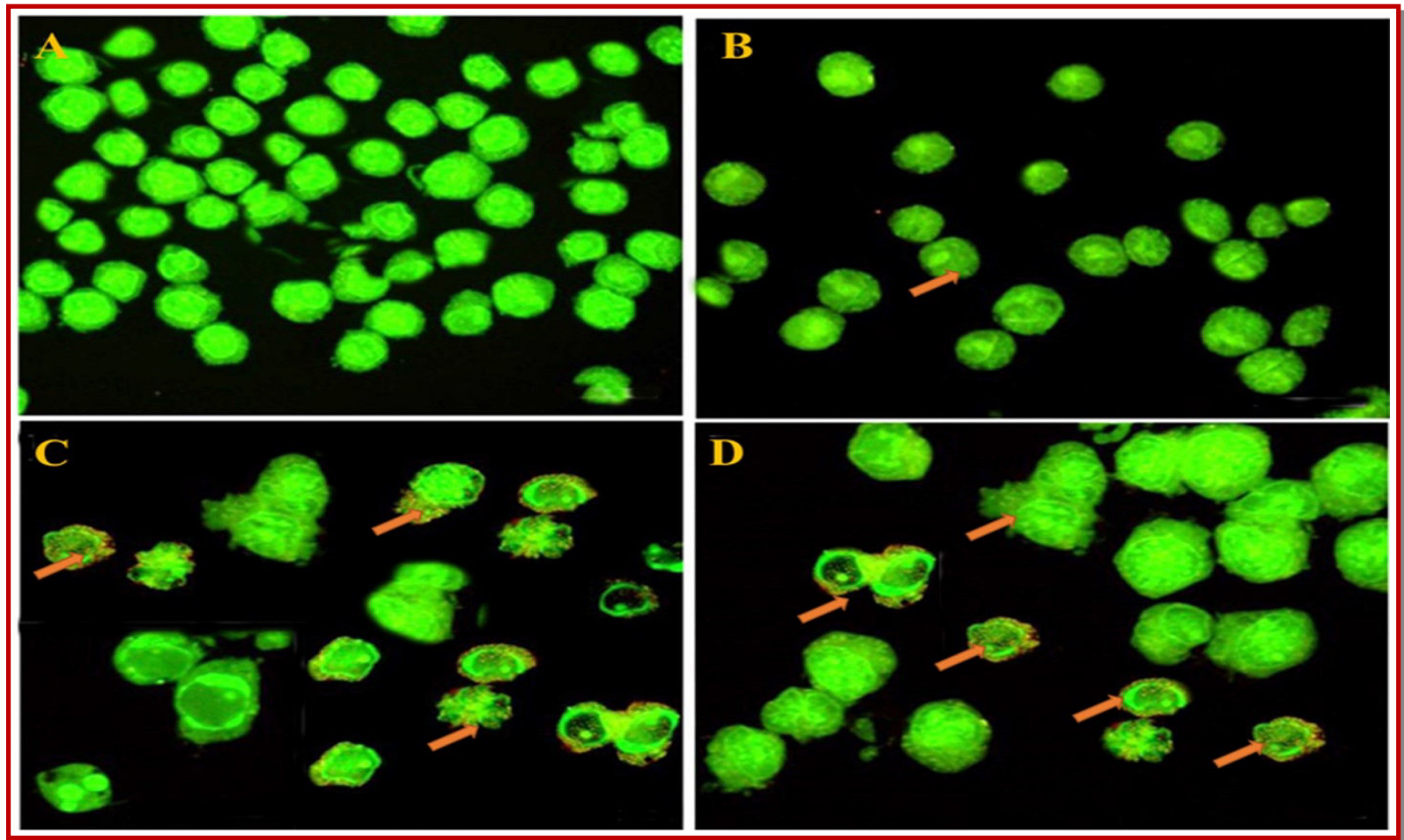

Figure 3: Fluorescent micrographs of acridine orange and propidium iodide double-stained A-549 cells. Intact green nuclei appear in control (A) conditions suggesting a good cell viability. After exposure to different doses of theaflavin-3-Gallate, apoptotic cells appear. A, shows control cells with normal cellular morphology. B, C and D represent cells treated with 20,80 and 160 $\mu \mathrm{M}$ dose of theaflavin-3-gallate

various concentrations of theaflavin-3-gallate for 48 hours several of the cells became rounded and shrunken, and detached from each other or floated in the medium.

\section{Study of apoptosis using acridine orange/propidium iodide double staining}

In control (Figure 3A) conditions, cells showed preserved green nuclei, indicating a good viability. However, after exposure to $20 \mu \mathrm{M}$ of theaflavin-3-gallate, late apoptotic and necrotic changes were observed (Figure 3B). However, 80 and $160 \mu \mathrm{M}$ of theaflavin-3-gallate induced apoptotic features including chromatin margination in cup-shaped masses and presence of micronuclei (Figure 3C, D).

Theaflavin-3-gallate induces G2/M phase cell cycle arrest in A-549 lung cancer cells

In order to demonstrate whether theaflavin-3-gallate induces cell cycle disturbances in A-549 cells, flow cytometric analysis using propidium iodide as a staining agent was performed after theaflavin-3-gallate treatment at different concentrations $(0,20,80$, and 160 $\mu \mathrm{M})$ for 48 hours. The population of cells in $\mathrm{G} 2 / \mathrm{M}$ phase increased from $2.7 \%$ (untreated control) to $6.8 \%$, $17.2 \%$ and finally to $46.5 \%$ after treatment with 20,80 and $160 \mu \mathrm{M}$ concentration of theaflavin-3-gallate respectively. The cells in the G0 phase also witnessed a slight increase from $2.1 \%$ to $4.6 \%, 12.3 \%$ and $18.7 \%$ after treatment with 20, 80 and $160 \mu \mathrm{M}$ concentration of theaflavin-3-gallate respectively (Figure 4A-D).

\section{Annexin V-FITC/PI assay for apoptosis evaluation and quantification}

Annexin V/propidium iodide double staining was used to detect apoptosis in the human lung cancer cells (A549) (Figure 5). A-549 cells were treated with different concentration $(0,20,80$ and $160 \mu \mathrm{M})$ of theaflavin-3gallate for 48 hours. Theaflavin-3-gallate induced both early and late apoptosis in a concentration-dependent manner (Figure 5B-D) as compared to the untreated control cells (Figure 5A). The different quadrants Q1, Q2, Q3 and Q4 represent necrotic cells, late apoptotic cells, viable cells and early apoptotic cell population respectively. Percentage of apoptotic cells increases from $5.2 \%$ in control cells (A), to $17.1 \%, 49.1 \%$ and 78.2 $\%$ in $20 \mu \mathrm{M}(\mathrm{B}), 80 \mu \mathrm{M}(\mathrm{C})$ and $160 \mu \mathrm{M}$ (D) theaflavin-3gallate-treated cells respectively.

Effects of theaflavin-3-gallate on the migration of A549 cells

In this experiment, we assessed the effect of theaflavin3-gallate on the cell migration in A-549 NSCLC cells. Confluent cells were scratched and then treated with theaflavin-3-gallate in a complete medium for 48 hours. The number of cells migrated into the scratched area was photographed $(\times 40)$ and calculated as a percentage (\%) of migration. As can be seen in Figure 6, theaflavin- 


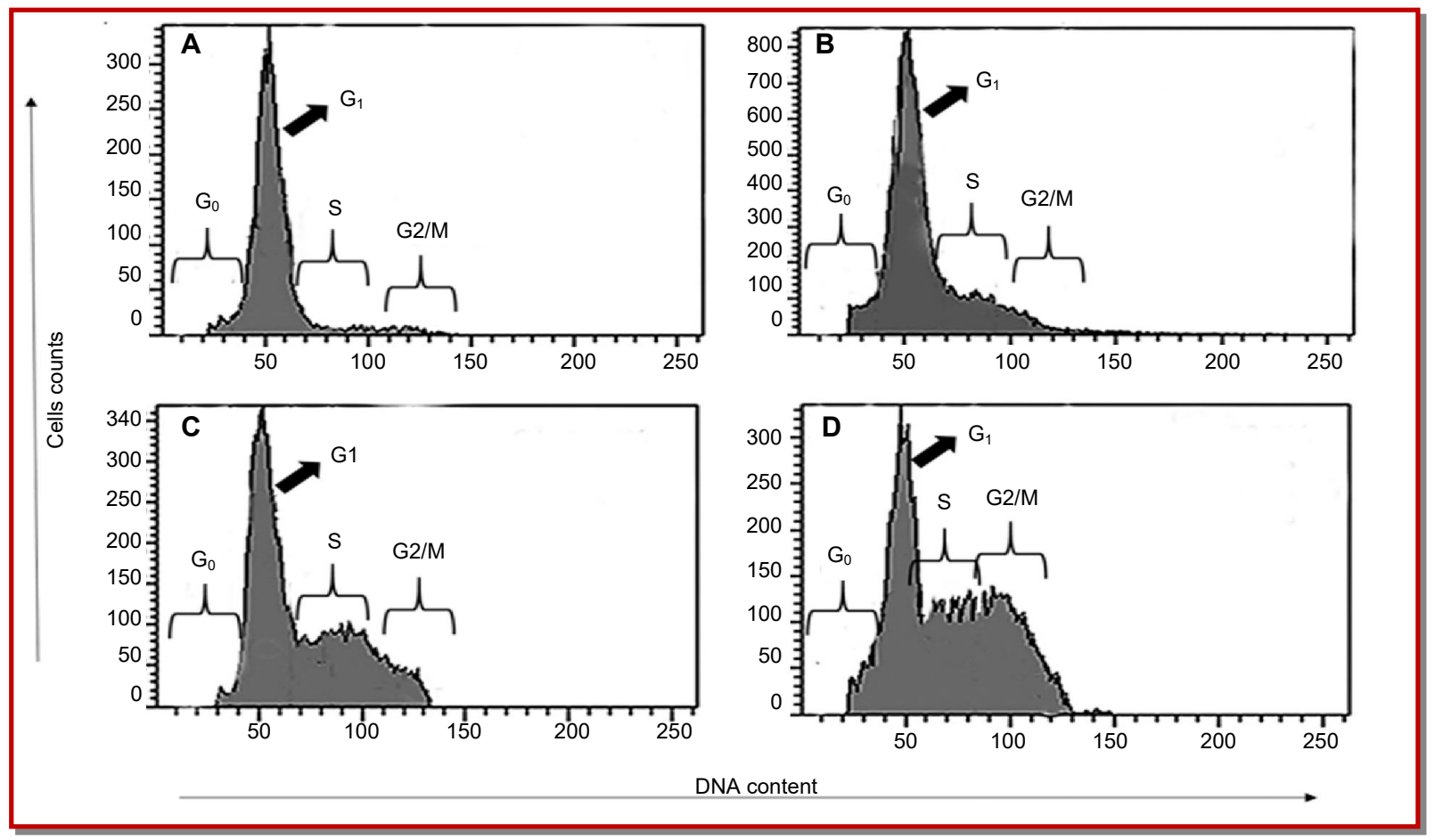

Figure 4: Effect of theaflavin-3-gallate on cell cycle phase distribution in A-549 non-small cell lung cancer cells. The cells were treated with different doses of the compound for 48 hours. The cell cycle distribution was determined by a flow cytometric analysis of the DNA content after staining with propidium iodide. A, shows control while as B, C and D represent cells treated with 20,80 and $160 \mu \mathrm{M}$ dose of theaflavin-3-gallate respectively

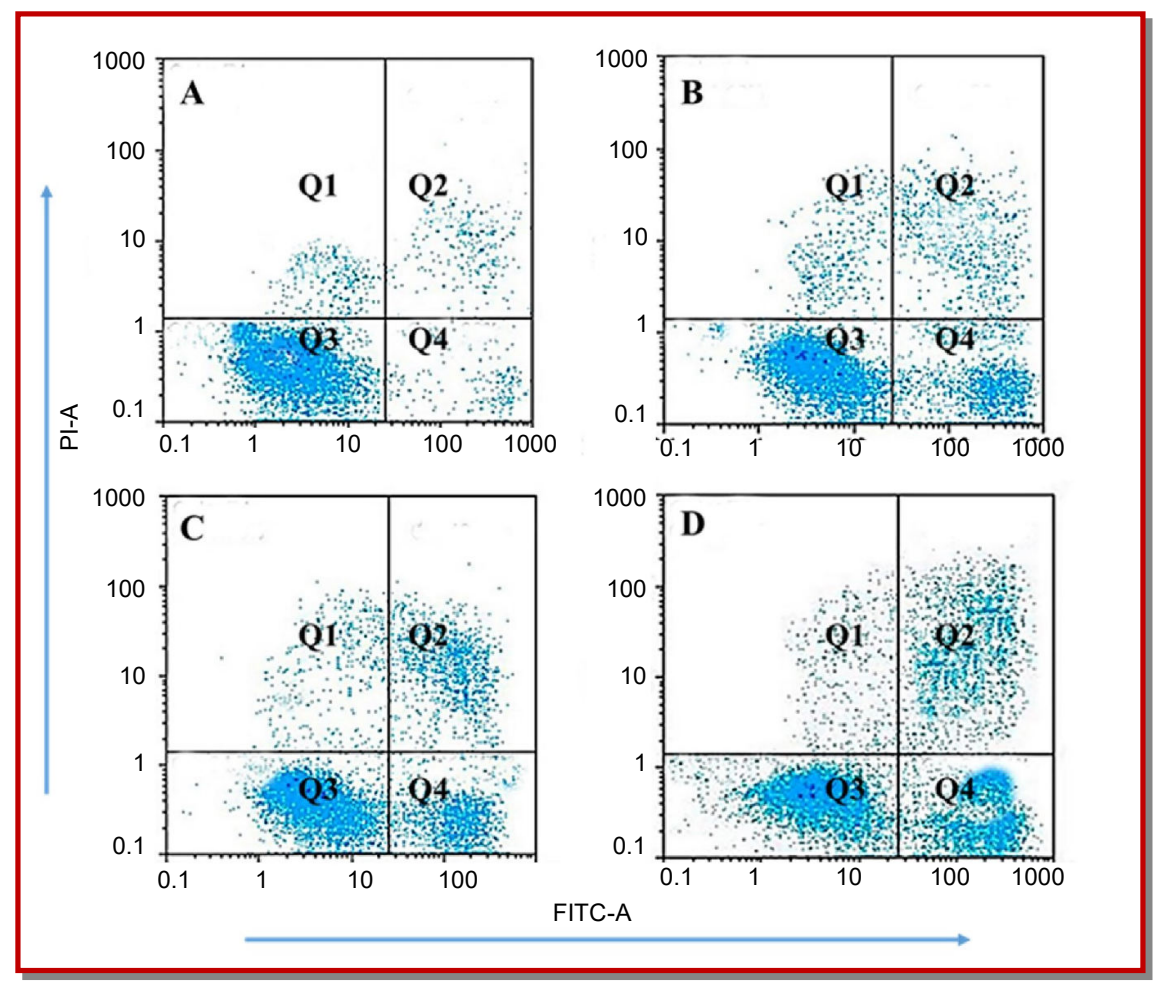

Figure 5: Quantification of theaflavin-3-gallate-induced apoptosis in human lung cancer cells (A-549). The cells were subjected to different doses of theaflavin-3-gallate $(0,20,80$ and $160 \mu \mathrm{M})$ for 48 hours and analyzed by flow cytometry with annexin V-FITC/ PI staining. The different quadrants Q1, Q2, Q3 and Q4 represent necrotic cells, late apoptotic cells, viable cells and early apoptotic cell population respectively. Percentage of apoptotic cells increases from $5.2 \%$ in control cells (A), to $17.1 \%, 49.1 \%$ and $78.1 \%$ in 20 $\mu \mathrm{M}(\mathrm{B}), 80 \mu \mathrm{M}(\mathrm{C})$ and $160 \mu \mathrm{M}(\mathrm{D})$ theaflavin-3-gallate-treated cells respectively 


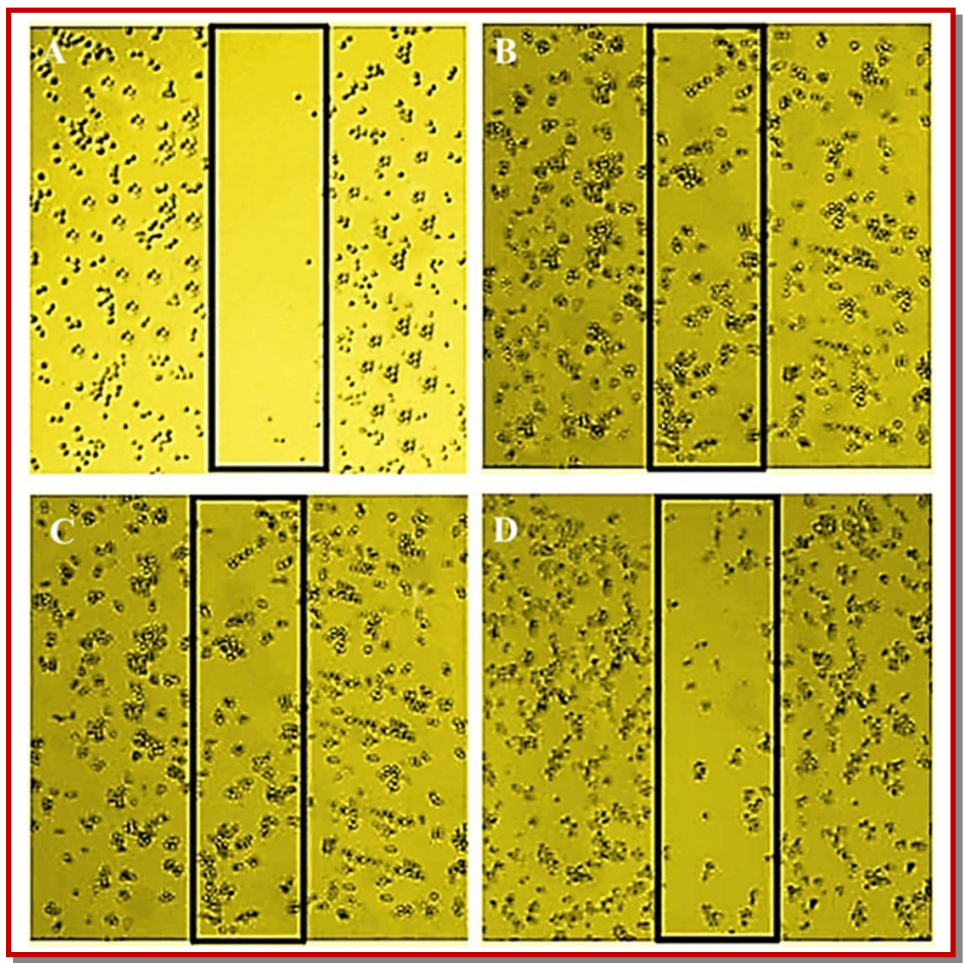

Figure 6: Inhibitory effects of theaflavin-3-gallate on the migration of A-549 cells. (A) Initial view of scratch at time 0, before any drug treatment, (B) control, after 24 hours, (C) theaflavin-3-gallate-treated cells at 24 hours at a dose of $80 \mu \mathrm{M}$, and (D) theaflavin-3gallate-treated cells at 24 hours at a dose of $160 \mu \mathrm{M}$

3-gallatedistinctly reduced A-549 cell migration in a concentration dependent manner. Figure $6 \mathrm{~A}$ represents untreated $(0 \mu \mathrm{M})$ control cells while Figure $6 \mathrm{~B}, \mathrm{C}$ and $\mathrm{D}$ represent effect of 20,80 and $160 \mu \mathrm{M}$ dose of theaflavin3-gallate respectively. Treatment of the cells with theaflavin3-gallate led to a decrease in the rate at which the cells migrate into the clear area created by scratch.

\section{Effects of theaflavin-3-gallate on the invasion of A-549 cells}

Lung cancer is very deadly because it is extremely invasive particularly in later stages. In this part of the study, we revealed whether theaflavin-3-gallate could inhibit the invasive behavior of NSCLC (A-549) cells. The invasive assay was designed using A-549 cells using matrigel-coated 24-well microchemotaxis chambers in the presence of theaflavin-3-gallate at various concentrations. As can be seen in Figure 7, theaflavin-3-gallate at various concentrations (ranging from $0 \mu \mathrm{M}$ to 20,80 and $160 \mu \mathrm{M}$ ) significantly inhibited the invasion of A-549 cells in a dose-dependent manner.

\section{Discussion}

Several natural products have been widely studied for their potential in cancer therapy and prevention over the last several decades. According to a recent review of natural products as sources of new drugs over the last 30 years, a total of $44(\sim 45 \%)$ out of 99 new anti-cancer drugs are either natural products or their synthetic or semisynthetic derivatives (Newman and Cragg, 2012). Paclitaxel (Taxol $\left.{ }^{\circledR}\right)$, for instance, is a plant-derived diterpenoid isolated from the bark of the Pacific yew tree, Taxus brevifolia. It is a potent inhibitor of cell division by disturbing normal microtubule breakdown during mitosis and is presently used as anti-mitotic chemotherapy for lung, breast, ovarian, head and neck cancer, as well as advanced forms of Kaposi's sarcoma (Wall and Wani, 1995). Even though conventional chemotherapeutic drugs induce cell death, they are inadequate due to their toxicity to normal cells. Identification of natural agents in the form of either plant extracts or a bioactive compound, which effectively exhibits apoptotic and cell cycle modulating properties and at the same time shows lesser toxicity to normal cells, is therefore crucial (Rebucci and Michiels, 2013).

Theaflavin-3-gallate is an antioxidant polyphenol which is regarded as the biologically important active components of black tea. According to a study, the antiproliferative and cytotoxic effects of theaflavin-3-gallateon Human squamous carcinoma (HSC-2) cells were more pronounced to the cancer cells than to the normal cells (Babich et al., 2008). Another study reports that theaflavin-3-gallateis effective in inhibiting the cancer cell proliferation of human liver cancer cells, gastric cancer cells and acute promyelocytic leukemia LH-60 cells (Tu et al., 2004). However, anticancer effect of theaflavin-3gallate on the A-549 non-small cell lung cancer cells has 


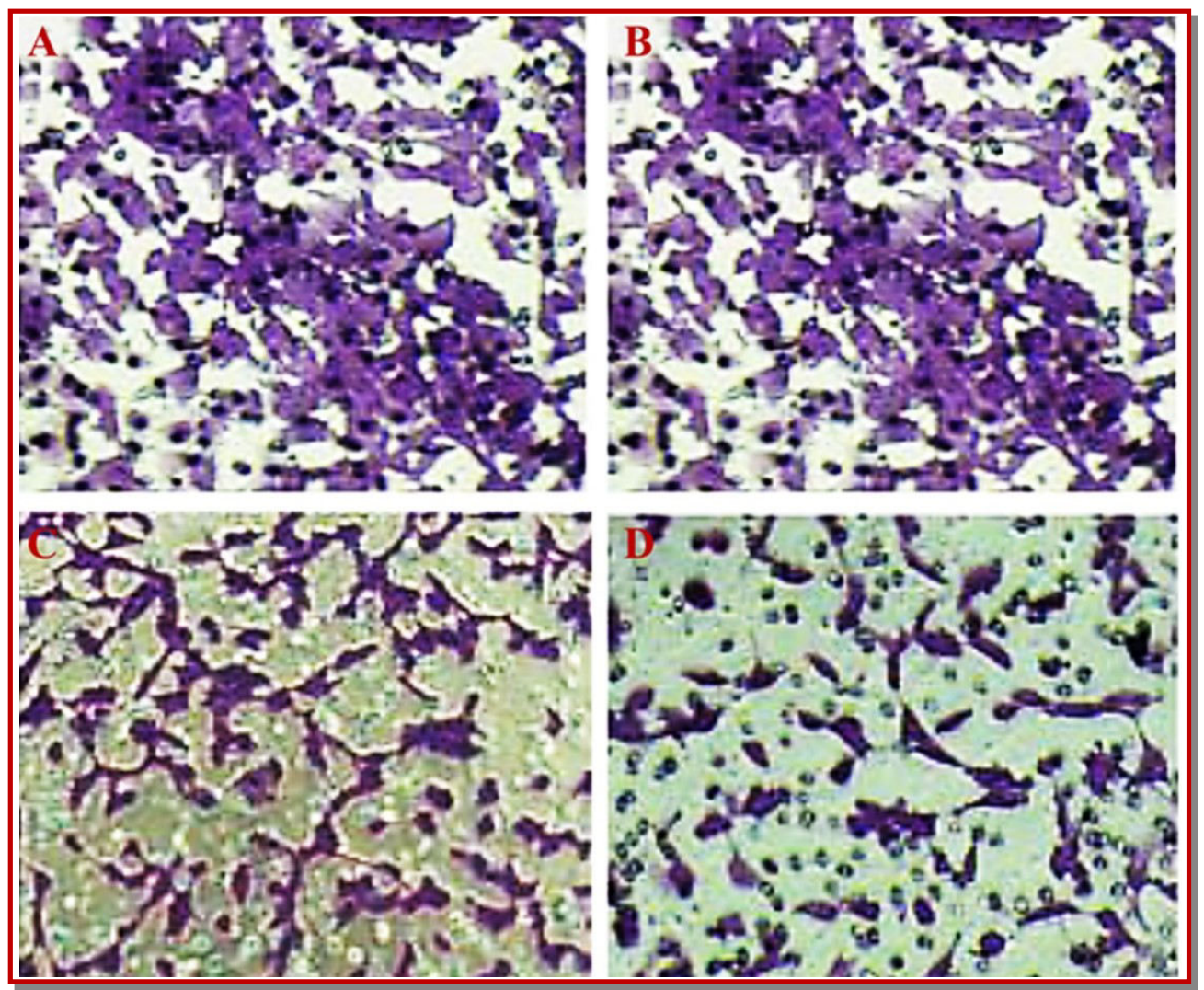

Figure 7: Theaflavin-3-gallate decreases invasion of A-549 non-small cell lung cancer cells. Invasion assay was carried out using modified 24-well microchemotaxis chambers. Then selected fields were photographed (x100), and the number of cells migrated to the lower surface was calculated as a percentage of invasion. A shows untreated normal cells, while as B, C and D represent effect of 20,80 and $160 \mu \mathrm{M}$ dose of theaflavin-3-gallate on cell invasion respectively

not been reported so far to the best of our knowledge. As such the aim of the current study was to investigate the effect of this polyphenolic antioxidant compound on the A-549 cancer cell growth. Theaflavin-3-gallate showed potent, concentration dependent as well as time-dependent growth inhibitory effects against A-549 cells. Phase contrast microscopy revealed that cells became rounded and shrunken, and detached from each other or floated in the medium after theaflavin-3gallate treatment.

In the progression of cancer, avoidance of apoptosis is one of the key factors resulting in overpopulation of malignant cells. Apoptosis is an active form of cell death directed by a set of prosurvival and antisurvival genes. There is a strong connection between loss of apoptotic control and cancer instigation and progression, as tumor cells lose their capacity to activate the death signaling pathway (Rebecca, 2011; Fulda and Debatin, 2003). Other than apoptosis, deregulated cellcycle control is a key feature of cancer development. Fluorescence microscopy showed that theaflavin-3gallate treatment triggered the appearance of late apoptotic and necrotic cells showing apoptotic features including chromatin margination in cup-shaped masses and micronuclei presence. Theaflavin-3-gallate also induced both early and late apoptosis in a concentration -dependent manner. The compound also disturbed cell cycle phase distribution inducing cell cycle arrest at G2/M phase of the cell cycle. Cancer cell migration and invasion were also significantly inhibited by the increasing doses of theaflavin-3-gallate.

\section{Conclusion}

The current findings demonstrate that theaflavin-3gallate can act as a lead natural product based anticancer agent owing to its tendency to inhibit lung cancer cell proliferation through the induction of apoptosis, cell cycle arrest and inhibition of cell migration and invasion.

\section{Conflict of Interest}

The authors declare that there is no conflict of interest to reveal.

\section{References}

Alberg AJ, Brock MV, Stuart JM. Epidemiology of lung cancer: 
Looking to the future. J Clin Oncol. 2005; 23: 3175-85.

American Cancer Society. Cancer Facts \& Figures 2014. Atlanta, American Cancer Society, 2014.

Babich H, Gottesman RT, Liebling EJ, Schuck AG. Theaflavin-3 -gallate and theaflavin-3'-gallate, polyphenols in black tea with prooxidant properties. Basic Clin Pharmacol Toxicol. 2008; 103: 66-74.

Drelichman A, Oldford J, Al-Sarraf M. Evaluation of cyclophosphamide, adriamycin, and cis-platinum (CAP) in patients with disseminated prostatic carcinoma: A phase II study. Am J Clin Oncol. 1985; 8: 255-59.

Fulda S, Debatin KM. Apoptosis pathways in neuroblastomatherapy. Cancer Lett. 2003; 197: 131-35.

Hanahan D, Weinberg RA. Hallmarks of cancer: The next generation. Cell. 2011; 144: 646-74.

Hann CL, Rudin CM. Management of small-cell lung cancer: Incremental changes but hope for the future. Oncology (Williston Park). 2008; 22: 1486-92.

Joyce JA, Pollard JW. Micro environmental regulation of metastasis. Nat Rev Cancer. 2009; 9: 239-52.

Liang CC, Park AY, Guan JL. In vitro scratch assay: A convenient and inexpensive method for analysis of cell migration in vitro. Nat Protoc. 2007; 2: 329-33.

Ma J, Dong C, Ji C. MicroRNA and drug resistance. Cancer Gene Therapy. 2010; 17: 523-31.

Mehlen P, Puisieux A. Metastasis: A question of life or death. Nat Rev Cancer. 2006; 6: 449-58.
Newman DJ, Cragg GM. Natural products as sources of new drugs over the 30 years from 1981 to 2010. J Nat Prod. 2012; 75: 311-35.

Raghavan D, Koczwara B, Javle M. Evolving strategies of cytotoxic chemotherapy for advanced prostate cancer. Eur J Cancer. 1997; 33: 566-74.

Rebecca SY. Apoptosis in cancer: From pathogenesis to treatment. J Exp Clin Cancer Res. 2011; 30: 87.

Rebucci M, Michiels C. Molecular aspects of cancer cell resistance to chemotherapy. Biochem Pharmacol. 2013; 85: 1219_ 26.

Sarkar FH, Banerjee S, Li Y. Pancreatic cancer: Pathogenesis, prevention and treatment. Toxicol Applied Pharmacol. 2007; 224: 326-36.

Trump DL, Marsh JC, Kvols LK, Citrin D, Davis TE, Hahn RG, Vogl SE. A phase II trial of carboplatin (NSC 241240) in advanced prostate cancer, refractory to hormonal therapy: An Eastern Cooperative Group Study. Invest New Drugs. 1990; 8: S91- 94.

Tu YY, Tang AB, Watanabe N. The theaflavin monomers inhibit the cancer cells growth in vitro. Acta Biochim Biophys Sin. 2004; 36: 508-12.

Wall ME, Wani MC. Camptothecin and taxol: Discovery to clinic-Thirteenth Bruce F. Cain Memorial Award Lecture. Cancer Res. 1995; 55: 753-60.

Yagoda A, Watson RC, Natale RB, Barzell W, Sogani P, Grabstald H, Whitmore WF. A critical analysis of response criteria in patients with prostatic cancer treated with cisdiamminedichloride platinum II. Cancer. 1979; 44: 1553-62. 